\title{
Quantitative Imaging in Muscle Diseases with Focus on Non-proton MRI and Other Advanced MRI Techniques
}

\author{
Marc-André Weber, MD, MSc ${ }^{10}$ \\ Mike P. Wattjes, MD, $\mathrm{PhD}^{6}$ \\ ${ }^{1}$ Institute of Diagnostic and Interventional Radiology, Pediatric \\ Radiology and Neuroradiology, University Medical Center Rostock, \\ Rostock, Germany \\ 2 Institute of Radiology, University Hospital Erlangen, Friedrich- \\ Alexander-Universität Erlangen-Nürnberg (FAU), Erlangen, Germany \\ ${ }^{3}$ Medical Physics in Radiology, German Cancer Research Center \\ (DKFZ), Heidelberg, Germany \\ ${ }^{4}$ C.J. Gorter Center for High Field MRI, Department of Radiology, \\ Leiden University Medical Center, Leiden, The Netherlands \\ ${ }^{5}$ Duchenne Center, The Netherlands \\ 6 Department of Diagnostic and Interventional Neuroradiology, \\ Hannover Medical School, Hannover, Germany
}

Armin M. Nagel, $\mathrm{PhD}^{2,30}$

Hermien E. Kan, $\mathrm{PhD}^{4,5}$

\begin{abstract}
Address for correspondence Marc-André Weber, MD, MSc, Institute of Diagnostic and Interventional Radiology, Pediatric Radiology and Neuroradiology, University Medical Center Rostock, ErnstHeydemann-Str. 6, Rostock D-18057, Germany (e-mail: marc-andre.weber@med.uni-rostock.de).
\end{abstract}

Semin Musculoskelet Radiol 2020;24:402-412.

\begin{abstract}
\section{Keywords}

- MR imaging

- muscle

- myopathies

- pattern recognition

- non-proton imaging

The role of neuromuscular imaging in the diagnosis of inherited and acquired muscle diseases has gained clinical relevance. In particular, magnetic resonance imaging (MRI), especially whole-body applications, is increasingly being used for the diagnosis and monitoring of disease progression. In addition, they are considered as a powerful outcome measure in clinical trials. Because many muscle diseases have a distinct muscle involvement pattern, whole-body imaging can be of diagnostic value by identifying this pattern and thus narrowing the differential diagnosis and supporting the clinical diagnosis. In addition, more advanced MRI applications including nonproton MRI, diffusion tensor imaging, perfusion MRI, T2 mapping, and magnetic resonance spectroscopy provide deeper insights into muscle pathophysiology beyond the mere detection of fatty degeneration and/or muscle edema. In this review article, we present and discuss recent data on these quantitative MRI techniques in muscle diseases, with a particular focus on non-proton imaging techniques.
\end{abstract}

Neuromuscular imaging is increasingly being used for the diagnostic work-up of patients with suspected acquired or inherited muscle diseases. ${ }^{1-3}$ Recent review articles stressed the importance of dedicated imaging modalities such as ultrasonography and magnetic resonance imaging (MRI) in the detection of muscle pathology (e.g., muscle edema or edema-like changes, fat replacement, and atrophy) to support the clinical diagnosis and aid further diagnostic steps, for example, guiding muscle biopsies. ${ }^{4-7}$ Modern medicine is precision and individualized medicine, meaning the correct therapy at the right moment for the right patient. For instance, diagnostic tests will guide clinical decision making to prescribe a specific drug, depending on the specific disease entity and the patient's prognosis as a responder or nonresponder to a given treatment. Moreover, precision medicine relies on the availability of robust, reliable, and validated molecular and other biomarkers such as imaging markers. Precision medicine was declared by President Barack Obama as a national task of the United States in his State of the Union Address on January 20, 2015.

\section{Large-volume MRI}

In the field of muscular diseases, the role of radiology facilitating precision medicine may lie in its potential to depict large volumes, for instance, the human being as a
Issue Theme Advanced Quantitative MSK Imaging; Guest Editors, Eric Y. Chang, MD and Edwin H.G. Oei, MD, PhD
Copyright $\odot 2020$ by Thieme Medical Publishers, Inc., 333 Seventh Avenue, New York, NY 10001, USA. Tel: +1(212) 760-0888.
DOI https://doi.org/ 10.1055/s-0040-1712955. ISSN $1089-7860$. 
whole. ${ }^{8}$ In the field of myopathies, whole-body (wb)-MRI may allow for the identification of individual patterns of muscle involvement, the extent of this involvement and risk stratification, detection of possible and clinically relevant comorbidities, as well as potentially radiomics-genomics applications. It has been demonstrated conclusively that neuromuscular imaging techniques can identify involvement patterns of muscle pathology based on the patterns of fat replacement, muscle edema, and atrophy that are to a certain degree specific for disease entities, coining the term "pattern recognition." In particular, the diagnostic value of this approach was demonstrated in the differential diagnosis of inherited muscle diseases. ${ }^{1,4,9}$ The wb-MRI protocols have been used to assess myopathies (with a very good correlation of fat replacement and paresis) for more than a decade and also to detect subclinical involvement. Examples of wb-MRI protocols even at higher magnetic field strengths are described elsewhere. ${ }^{10-12}$

\section{Focused and Quantitative MR Imaging}

An important role of radiology in precision medicine may be high-resolution imaging or imaging using advanced/ quantitative techniques offering information about microstructural tissue damage and even the metabolism on a cellular level allowing a correlation with muscle function and in particular the genotype-phenotype relationship. In addition to the use of semiquantitative visual rating scales, quantitative muscle MRI techniques (qMRI) can assess the degree of muscle degeneration and inflammation more precisely. This was exemplified by the European Union funding of the Cooperation in Science and Technology (COST Association) action BM1304 called MYO-MRI (www.myo-mri.eu), in which development and protocol sharing of quantitative imaging techniques was one of the goals. ${ }^{13}$

Moreover, several review articles have been published on various qMRI techniques in recent years. ${ }^{13-16}$ In particular, qMRI measures are valuable for monitoring disease progression. ${ }^{1,3,7,12,13,17,18}$ The detection of subclinical disease progression, especially for those muscles that are not easily accessible based on clinical examination, the monitoring of the natural history of disease, and checking potential therapeutic effects by using qMRI may be especially valuable in clinical trials. ${ }^{13,19}$

One particular qMRI application is the Dixon technique, or multiecho chemical shift encoded water-fat imaging. ${ }^{20}$ Quantitative MRI to measure the fat replacement of skeletal muscle by either chemical shift encoded imaging methods like Dixon or iterative decomposition of water and fat with echo asymmetry and least-squares estimation (IDEAL), or magnetic resonance spectroscopy (MRS) was demonstrated to provide a sensitive objective end point in several studies in muscular dystrophies. ${ }^{21}$ Using these chemical shift encoded water-fat imaging sequences, the muscle fat fraction (MFF) can be calculated by positioning regions of interest (ROIs) on different muscles on the MR images. After conducting ROI analysis on both water ("w") and fat ("f") images, the MFF can be calculated in this equation as $\mathrm{MFF}=\mathrm{f} /(\mathrm{f}+\mathrm{w}){ }^{21,22}$

Recent data suggest that the role of neuromuscular imaging goes far beyond the diagnostic stage and includes monitoring of disease progression as well as treatment efficacy and safety monitoring. ${ }^{23,24}$ A large number of studies showed a good to very good correlation of MFF with strength and function measures cross-sectionally in a wide range of muscle diseases, ${ }^{12,19,25-28}$ and recently MFF was even shown to be able to predict future function in Duchenne muscular dystrophy (DMD). ${ }^{29,30}$ This represents an important step forward in the road to a surrogate end point in clinical trials. ${ }^{13,31}$

A second approach for the reliable assessment of degenerative disease activity in muscle tissue including fatty degeneration represents T2 mapping of skeletal muscle. T2 mapping can be performed without accounting for fat replacement (global T2) and by removing the fat during postprocessing or by using MRS (water T2). ${ }^{13,23}$ Other promising techniques may also play a role, for instance diffusion tensor imaging (DTI), ${ }^{31} \mathrm{P}$ MRS, and non-proton MRI such as ${ }^{35} \mathrm{Cl}$ and ${ }^{23} \mathrm{Na}$ MRI.

Although several review articles have appeared in the last decade on muscle imaging, the focus of these articles was largely on proton MRI and MRS. ${ }^{9,13,15,21}$ Thus our focus is an overview of qMRI in muscle diseases with a particular focus on non-proton MRI.

\section{Non-proton MRI}

The increasing availability of high-field MRI systems $\left(B_{0} \geq 3 \mathrm{~T}\right)$ enables MR imaging of non-proton nuclei (e.g., ${ }^{23} \mathrm{Na},{ }^{35} \mathrm{Cl},{ }^{39} \mathrm{~K}$, and ${ }^{17} \mathrm{O}$ ) in clinically feasible measurement time and acceptable spatial resolution. ${ }^{32,33}$ Among all non-proton nuclei, sodium $\left({ }^{23} \mathrm{Na}\right)$ exhibits the best properties for in vivo applications. However, the in vivo signal-to-noise ratio (SNR) of ${ }^{23} \mathrm{Na}$ MRI is still four orders of magnitude lower compared with the SNR of ${ }^{1} \mathrm{H}$ MRI. This difference is caused by the low in vivo concentration and the $\sim 10$-fold lower MR sensitivity of the ${ }^{23} \mathrm{Na}$ nucleus. In addition, the ${ }^{23} \mathrm{Na}$ MRI signal of skeletal muscle tissue experiences fast biexponential transverse relaxation $\left(\mathrm{T}_{2 \mathrm{f}}{ }^{*} \sim 1.5-2.5 \mathrm{~ms}\right.$; $\left.\mathrm{T} 2 \mathrm{~s}^{*} \sim 15-30 \mathrm{~ms}\right){ }^{34}$ Thus ultrashort echo time pulse sequences ( $\mathrm{TE}<1 \mathrm{~ms}$ ) are required for quantitative imaging. Because the $\mathrm{T} 1$ relaxation time of the sodium nucleus within the muscle tissue is short $(12-25 \mathrm{~ms}),{ }^{34}$ rapid averaging can be used to improve the SNR of ${ }^{23} \mathrm{Na}$ MRI. Therefore, ${ }^{23} \mathrm{Na}$ MRI can depict changes of muscular sodium $\left(\mathrm{Na}^{+}\right)$concentration in sodium muscle channelopathies and other rare muscle channelopathies and dystrophies, such as hypokalemic periodic paralysis and DMD. ${ }^{13,35}$ It was shown that quantitative ${ }^{23} \mathrm{Na}$ MRI measurements can be performed with good repeatability and reproducibility, which is important for longitudinal studies and future multicenter trials. ${ }^{36}$

\section{Applications of ${ }^{23} \mathrm{Na}$ MRI in Muscular Channelopathies and Muscle Dystrophies}

Myotonia is an involuntary slowed relaxation after a forceful voluntary muscle contraction leading to the clinical presentation of muscle stiffness. The main feature of myotonia is the electrical hyperexcitability of the muscle fiber membrane. 
During repeated contractions the stiffness classically recedes, coining the term "warm-up" phenomenon. In contrast, patients with paradoxical myotonia (synonym: paramyotonia) experience worsening of muscle stiffness after repeated contractions or during cooling. Myotonia patients presenting with episodes of flaccid limb muscle weakness receive the diagnosis periodic paralysis. They present with episodic weakness spelling with varying intervals of normal muscle function. The major pathophysiologic hallmark is the electrical inexcitability of the muscle fiber membrane.

We can differentiate between two dominant episodic types of weakness with or without myotonia based on the serum $\mathrm{K}^{+}$level during the attacks of tetraplegia: hyperkalemic periodic paralysis (HyperPP) and hypokalemic periodic paralysis (HypoPP). Independently of the severity and frequency of the paralytic episodes, many patients develop a chronic progressive myopathy in their 40s, an age at which the attacks of weakness decrease. ${ }^{37,38}$ Although channelopathies including myotonias and periodic paralyses are clinically characterized by episodic symptoms, most patients present with progressive focal or general muscular weakness. Routine proton $\left({ }^{1} \mathrm{H}\right)$ MRI applications show normal muscle tissue morphology or demonstrate edematous or lipomatous changes in terms of fat replacement, atrophy, or even exercise-induced hypertrophy or myopathic pseudohypertrophy. ${ }^{38}$ Of note, none of these changes are specific for any particular disease entity. ${ }^{39}$

Muscle channelopathies can serve as proof-of-principle diseases for the use of ${ }^{23} \mathrm{Na}$ MRI. ${ }^{38}$ Physiologically, the intracellular $\mathrm{Na}^{+}$content is $\sim 10$ times lower than the extracellular concentration. This gradient is mandatory for the functioning of cells. The energy-consuming $\mathrm{Na}^{+}-\mathrm{K}^{+}$-ATPase contributes to this concentration gradient and helps maintain the membrane potential of cells. While pumping three sodium ions to the extracellular space, it carries only two potassium ions to the intracellular space of the cell. Thus in total it removes one positively charged ion from the intracellular space of the cell, causing a negatively charged cell membrane potential. A breakdown of the $\mathrm{Na}^{+}-\mathrm{K}^{+}$-ATPase or an increased open probability of mutated cellular ion channels (e.g., in muscular channelopathies ${ }^{37}$ ) results in an increase of the intracellular $\mathrm{Na}^{+}$concentration and subsequently to a depolarization of the cells.

Paramyotonia congenita (PC) is a channelopathy characterized by an increased intracellular $\mathrm{Na}^{+}$concentration, and cell depolarization can be easily and reproducibly provoked, for instance by exposure to cold and muscle load. The exposure to cold temperatures can trigger a long-lasting depolarizing $\mathrm{Na}^{+}$ inward current and muscle weakness. ${ }^{37}$ A potential provocation scenario is cooling for 25 minutes directly followed by a short exercise ( 2 minutes) consisting of dorsiflexion of the feet against resistance and standing on tiptoes. ${ }^{38}$ The provoked muscle of the PC patients shows a pronounced decrease of muscle strength and an increase of the ${ }^{23} \mathrm{Na}$ MRI signal. ${ }^{38}$ In contrast, ${ }^{1} \mathrm{H}$ MRI does not show any pathologic findings. Different ${ }^{23} \mathrm{Na}$ MR sequences are available. Among these, the ${ }^{23} \mathrm{Na}$ inversion recovery MRI provides a strong weighting toward intracellular sodium and can visualize this increase of the intracellular $\mathrm{Na}^{+}$concentration (-Fig. 1). ${ }^{38,40}$
One clinical example demonstrating the value of qMRI is ${ }^{23} \mathrm{Na}$ MRI for the evaluation and monitoring of muscular channelopathies, in which an autosomal dominant bequeathed defect of muscular $\mathrm{Na}^{+}$channels leads to a pathologic $\mathrm{Na}^{+}$influx and to intermittent or permanent muscular paresis as well as muscular stiffness. ${ }^{23} \mathrm{Na}$ MRI by which aspects of muscular pathogenesis such as muscular $\mathrm{Na}^{+}$homeostasis can be visualized and monitored has conclusively demonstrated the added value of qMRI techniques in the diagnosis and monitoring of muscular $\mathrm{Na}^{+}$channel diseases because ${ }^{23} \mathrm{Na}$ MRI is able to depict an intracellular muscular sodium accumulation in close correlation to clinical symptoms, such as the development of muscular paresis. This sodium accumulation correlates well with the severity of paresis, and this marker is reproducible. ${ }^{38}$ The key points can be summarized as follows ${ }^{38}$.

1. Muscular ion channelopathies include the non-dystrophic myotonias and periodic paralyses. The common feature is the alteration of the muscle membrane potential due to changes in ion conductivities. This implies that gradients for $\mathrm{Na}^{+}$and $\mathrm{Cl}^{-}$are different, leading to an alteration of the membrane excitability. Clinical manifestations of these muscular diseases are myotonia or weakness associated with hyper- or hypokalemia.

2. Periodic paralysis occurs episodically with varying intervals of normal muscle function because the underlying ion channel defects are usually well compensated. An additional trigger, for instance, exposure to cold, is often required for channel malfunction and thus triggers clinical symptoms.

3. Muscle channelopathies can be diagnosed and monitored by qMRI techniques for demonstration of the pathogenesis, estimating prognosis, and monitoring treatment.

4. For example, ${ }^{23} \mathrm{Na}$ MRI can depict an intracellular muscular sodium accumulation simultaneous to development of muscular paresis in paramyotonia and HyperPP and HypoPP. The sodium accumulation correlates well with clinical manifestations such as the grade of paresis, and the measurements are reproducible.

5. In HyperPP and HypoPP, treatment schemes that are antiedematous have the potential to alleviate muscular edema and weakness.

\section{Sodium Concentration, Edema, and Muscle Strength} HypoPP is characterized by episodes of flaccid skeletal muscle weakness. These episodes are accompanied by a drop in serum potassium. HypoPP is an autosomal-dominant neuromuscular disorder. Muscle strength is mostly reduced in muscles recently exercised. The episodes of muscle weakness usually last hours but may persist for days. Some patients may develop a progressive proximal myopathy with seemingly permanent weakness. ${ }^{41,42}$ A correlation between the frequency of weakness episodes and subsequent muscle degeneration has not been observed. In most patients, HypoPP is caused by mutations in one of two genes, CACNA1S and SCN4A. Both encode voltage-gated ion channels of skeletal muscle, namely, Cav1.1, the tubular L-type calcium channel, and Nav1.4, the sarcolemmal sodium channel. The mutations are localized in a 


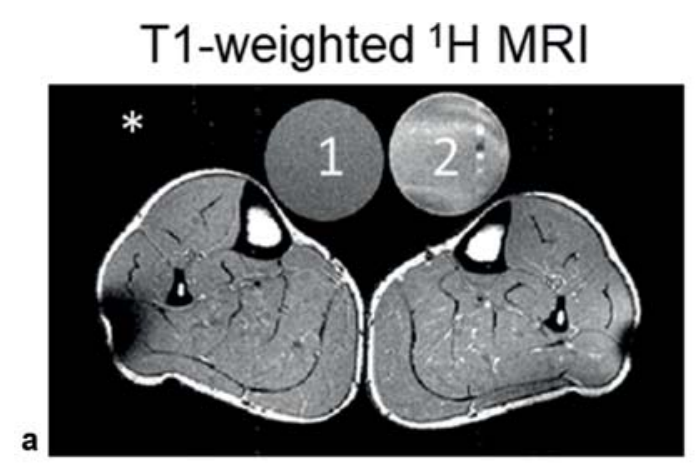

${ }^{23} \mathrm{Na}$ IR MRI
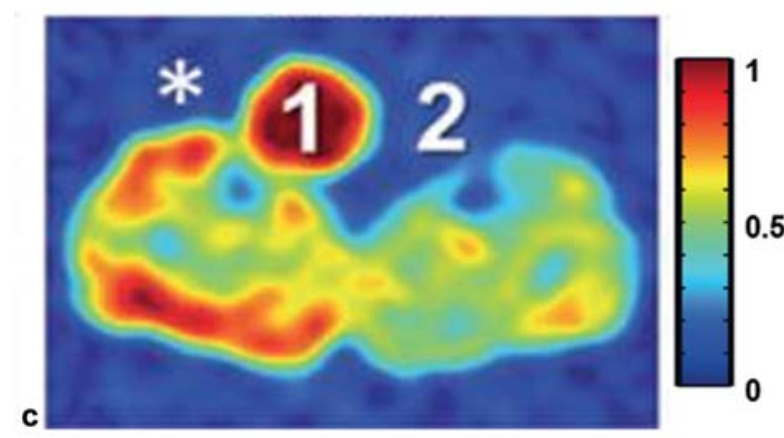

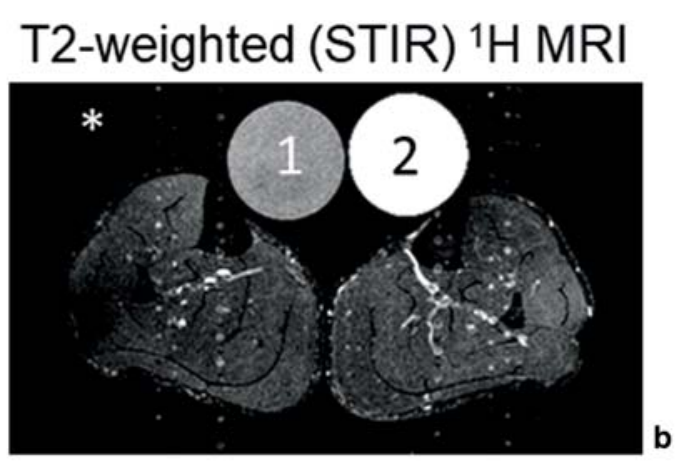

${ }^{23} \mathrm{Na} \mathrm{MRI}$

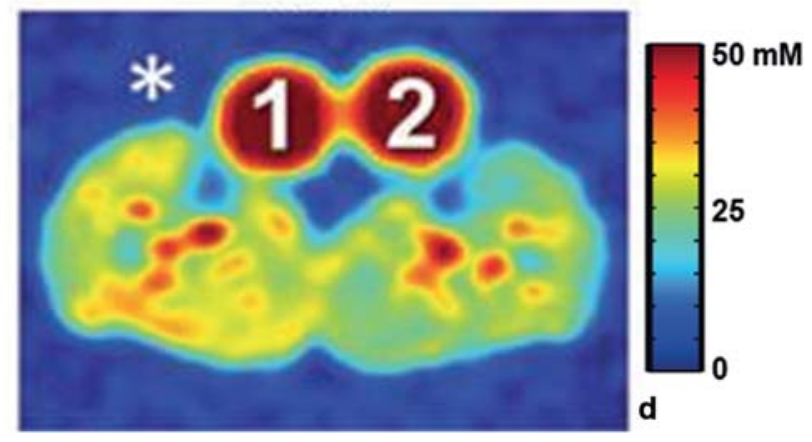

Fig. 1 The ${ }^{1} \mathrm{H}$ and ${ }^{23} \mathrm{Na}$ magnetic resonance (MR) images of a patient with a muscular channelopathy (paramyotonia congenita). The right lower leg (asterisk) was cooled with an ice bag, resulting in an increase of the intracellular $\mathrm{Na}^{+}$concentration and in severe muscle weakness. Two reference tubes were used for signal normalization (1: $51.3 \mathrm{mmol} / \mathrm{L} \mathrm{NaCl}$ and 5\% agarose gel; $2: 51.3 \mathrm{mmol} / \mathrm{L} \mathrm{NaCl}$ solution). (a) T1-weighted and (b) T2-weighted fat-suppressed ${ }^{1} \mathrm{H}$ MRI revealed no pathologic signal differences between the cooled (asterisk) and the non-cooled muscle. (c) The ${ }^{23} \mathrm{Na}$ inversion recovery (IR) MRI revealed a distinct increase of the signal in the cooled leg and thus visualizes the increase of the intracellular $\mathrm{Na}^{+}$content. Note: Reference tube 2 (pure saline solution) shows no signal intensity on ${ }^{23} \mathrm{Na}$ IR MRI. (d) The cooled leg shows a slightly increased total $\mathrm{Na}+$ concentration. STIR, short tau inversion recovery. (Reproduced with modifications from Nagel et al. ${ }^{40}$ )

transmembrane helical S4 segment thought to act as a voltage sensor and neutralize a positive amino acid such as arginine $(\mathrm{R})$ (Cav1.1; Nav1.4). Substitution of the $\mathrm{R}$ residue with histidine $(\mathrm{H})$ or glycine $(\mathrm{G})$ leads to a pathologic so-called gating pore current through the S4 segment that depolarizes the muscle and thus probably causes the weakness. ${ }^{42,43}$ Carbonic anhydrase inhibitor (CAI) therapy, especially with acetazolamide, has been the most common treatment choice for HypoPP in the past decades. ${ }^{44}$ In addition to genotyping and the response to therapy, muscle qMRI is a promising method to investigate the underlying cause of the muscle weakness. ${ }^{45}$

Muscular $\mathrm{Na}^{+}$concentration has been quantified by using 1.5-T, ${ }^{41} 3-\mathrm{T},{ }^{40,46}$ and $7-\mathrm{T}{ }^{23} \mathrm{Na}$ MRI (-Fig. 2). ${ }^{22,47} \mathrm{~A}$ recent observation of one female patient presenting with HypoPP over a period of 11 years revealed a permanent recovery from permanent muscle weakness by spironolactone plus $\mathrm{K}^{+}$ treatment. ${ }^{48}$ In addition, the therapeutic response of muscle tissue could be monitored with ultrasound, ${ }^{1} \mathrm{H}$ MRI, and ${ }^{23} \mathrm{Na}$ MRI. ${ }^{48}$ The patient was wheelchair bound at 8 years of age and yet remarkably regained ambulation when placed on spironolactone plus $\mathrm{K}^{+}$at age 18 , and she has retained nearnormal strength over the intervening 11 years. ${ }^{48}$ Ultrasound and ${ }^{1} \mathrm{H}$ MRI demonstrated muscle edema without any fat replacement or atrophy before treatment initiation that subsequently resolved when the muscle strength improved. $\mathrm{A}^{23} \mathrm{Na} \mathrm{MRI}$ revealed intracellular $\mathrm{Na}^{+}$overload, which was initially very severe with $\sim 50 \mathrm{mM}$ compared with normal values of $20 \mathrm{mM}$, but it then resolved and did not reoccur during pharmacotherapy. ${ }^{48}$ This longitudinal observation of a single patient demonstrated several interesting features of the periodic paralysis HypoPP:

1. The diagnosis could be delayed for more than a decade when the presentation is atypical with so-called permanent myopathic features rather than episodic attacks of weakness. This may occur especially when there is no family history for a de novo mutation.

2. Moderate to severe weakness that has persisted for a decade in HypoPP can be reversed with appropriate therapy.

3. Muscle imaging and molecular genetics are important diagnostic tools and could monitor reversal of chronic weakness with spironolactone plus $\mathrm{K}^{+}$. Normalization of image-based pathologic features like edema and intracellular $\mathrm{Na}^{+}$overload, as well as the absence of others (fat replacement or muscle atrophy), were associated with reversibility of clinical features.

4. Continued adequate therapy may preserve muscle structure and strength in the long term. However, weakness due to fat replacement could be considered progressive and irreversible. ${ }^{48}$

\section{Sodium MRI in Duchenne Muscular Dystrophy}

In addition to muscular channelopathies, several studies also reported the value of ${ }^{23} \mathrm{Na}$ MRI for muscle dystrophies such as DMD. ${ }^{35,45,49-51}$ In a 2012 study including eight patients 


\section{${ }^{7-T}{ }^{23} \mathrm{Na}$ IR MRI}
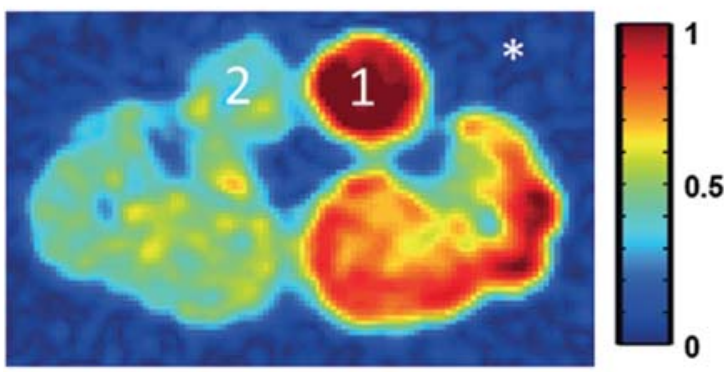

${ }^{3-T}{ }^{23} \mathrm{Na}$ IR MRI
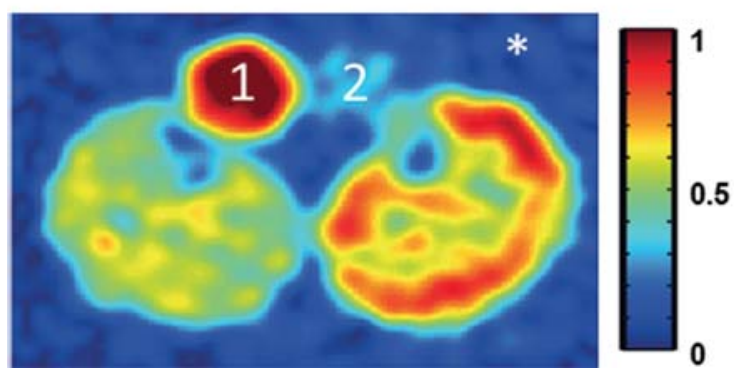
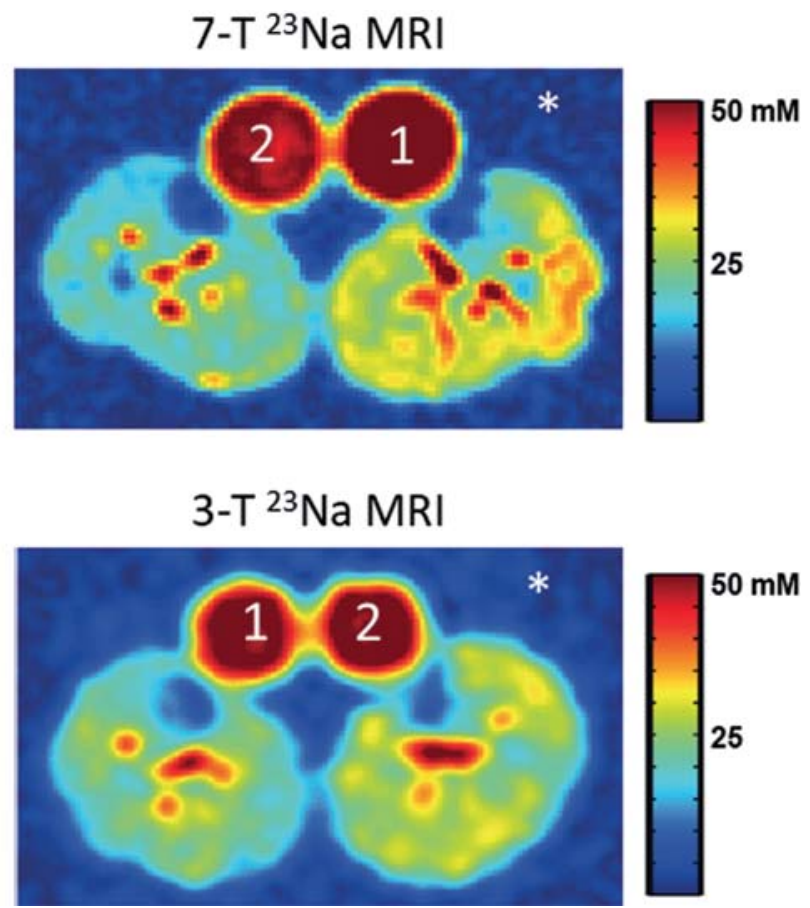

Fig. 2 Comparison of ${ }^{23} \mathrm{Na}$ magnetic resonance (MR) images obtained with a 7-T unit and on the same day with a 3-T unit of both lower legs in a 24-year-old man with paramyotonia congenita and full muscle strength before provocation. The left lower leg (asterisk) was cooled with an ice bag wrapped around the lower leg for 25 minutes. Directly after cooling, the patient had to dorsiflect his feet against resistance and stand on his tiptoes. The contralateral leg was not cooled and served as the reference. The ${ }^{23} \mathrm{Na}$ MR images demonstrate substantial increase of the intracellular $\mathrm{Na}^{+}$concentration that parallels muscle weakness of the left lower leg. Two reference tubes were used for signal normalization (1: $51.3 \mathrm{mmol} / \mathrm{L} \mathrm{NaCl}$ and $5 \%$ agarose gel; $2: 51.3 \mathrm{mmol} / \mathrm{L} \mathrm{NaCl}$ solution). In contrast to the total $\mathrm{Na}^{+}$concentration sequence (right column), the ${ }^{23} \mathrm{Na}$ inversion recovery (IR) sequence (left column) better reveals the distinct increase of the signal in the provoked leg and thus the increase of the intracellular $\mathrm{Na}^{+}$. Moreover, the higher field strength yields a better contrast-to-noise ratio at the same acquisition time.

with DMD and eight volunteers (both with a mean age of 9.5 years) and using $3-\mathrm{T}^{1} \mathrm{H}$ and ${ }^{23} \mathrm{Na}$ MR sequences, the muscular tissue $\mathrm{Na}^{+}$concentration was markedly increased in DMD $(38 \pm 6 \mathrm{mmol} / \mathrm{L})$ and remained constant during follow-up ( $n=7$, first: $38 \pm 6 \mathrm{mmol} / \mathrm{L}$; second: $37 \pm 4 \mathrm{mmol} / \mathrm{L}$ ). ${ }^{49}$ Muscular edema and fat content were also significantly elevated in DMD compared with healthy individuals.

Based on these data, it was concluded that ${ }^{23} \mathrm{Na}$ MRI demonstrated a muscular $\mathrm{Na}^{+}$overload in DMD. This permanent $\mathrm{Na}^{+}$overload in all DMD patients may be osmotically relevant and may play a role in the development of the muscle edema that was present and persisted in all studied DMD patients. ${ }^{35,38,45,49}$ This muscle edema may represent inflammation related to progressive muscle degeneration, but the previously described findings point to the suggestion that increased sodium concentrations and water precedes the dystrophic process and persists until fat replacement is complete. Thus muscle cell edema may represent the initial stage of muscular degeneration because muscular $\mathrm{Na}^{+}$overload was also reported in the chronic HypoPP. ${ }^{42}$

Of note, the prevailing theory of muscle edema in DMD is that this represents an inflammatory first stage of the disease with ongoing muscle degeneration. ${ }^{52}$ Further studies have investigated whether these increased sodium concentrations are relevant for pathogenesis and disease progression as well as whether this may be a potential target for treatment. ${ }^{53}$ The effects of various diuretic drugs on a cell model of DMD were investigated, demonstrating that both CAI and aldosterone antagonists could repolarize depolarized muscle fibers. CAI therapy is known to have acidifying effects that may be harmful to the ventilation of DMD patients. Therefore, research studies mainly concentrated on the modern spironolactone derivative eplerenone with a very high repolarizing power. ${ }^{53}$ In a pilot study, this drug was administered to a 22-year-old female DMD patient who was wheelchair dependent and did not receive any prior corticosteroid therapy. Eplerenone decreased both cytoplasmic sodium and water overload leading to an increase in muscle strength and mobility. This led to the conclusion that eplerenone might have some beneficial effect on DMD muscle tissue and that the cytoplasmic edema is rather cytotoxic and should be treated before fatty degeneration takes place. ${ }^{53}$

Moreover, it was reported that a low-dose application of a mild diuretic substance such as eplerenone is beneficial on the muscle tissue compared with glucocorticoid therapy in the early stages of two 7-year old DMD patients measured by ${ }^{23} \mathrm{Na}$ and ${ }^{1} \mathrm{H} \mathrm{MRI}$, myometry, and clinical testing before and after 1,3 , and 6 months of therapy with eplerenone or cortisone, respectively. ${ }^{50}$ During eplerenone therapy the authors detected a reduction of muscular edema and muscular $\mathrm{Na}^{+}$concentration that was more pronounced compared with the patient treated with cortisone. Myometricmeasured tissue parameters such as muscle stiffness had a 
more pronounced effect in the child treated with eplerenone after a first increase in muscle stiffness both after eplerenone and cortisone treatment. With the help of advanced quantitative muscle MRI, the hypothesis could be raised that eplerenone might be a possible new therapy option in DMD. In addition, it was shown that, in DMD, increased $\mathrm{Na}^{+}$concentrations can be present even in the absence of fatty degenerative changes or increases of the water T2 relaxation time. ${ }^{51}$ This demonstrates that ${ }^{23} \mathrm{Na}$ MRI can be a very sensitive technique for the analysis of muscular diseases.

\section{Genotype-Phenotype Correlation}

Altered $\mathrm{Na}^{+}$and chloride $\left(\mathrm{Cl}^{-}\right)$homeostasis can be visualized in periodic paralyses, where the resting membrane potential is reduced, ${ }^{37}$ by using $7-\mathrm{T}{ }^{23} \mathrm{Na}$ and ${ }^{35} \mathrm{Cl}$ MRI. ${ }^{22}$ Genetic alterations that affect muscle ion channels are linked to the rare but expanding group of inherited muscle channelopathies. ${ }^{54}$ These genetic mutations result in either an increase or a decrease in muscle membrane excitability. This leads to a variety of related clinical diseases:

1. Non-dystrophic myotonias show delayed relaxation after muscle contraction causing muscle stiffness and pain.

2. Periodic paralyses are characterized by episodes of flaccid muscle paralysis with intervals of normal muscle function. $^{54}$

The disease of muscle channelopathies is provoked by mutations in calcium, chloride, potassium, and sodium ion channels. The mutations result in decreased or increased muscle membrane excitability. This is due to altered muscle membrane potential related to changes in ion conductivities. The early and accurate diagnosis of channelopathies is clinically highly relevant because treatment and management strategies differ based on mutation and clinical phenotype. $^{54}$ As mentioned earlier, cation leaks play an important role in HypoPP and cause clinical manifestations in terms of paralytic attacks. ${ }^{42}$ Andersen-Tawil syndrome (ATS) is a rather exceptional disease in the spectrum of channelopathies because it is a multisystem disorder. Clinically, ATS is characterized by periodic paralysis, ventricular arrhythmias, and dysmorphic features. ${ }^{55}$ Most ATS patients present with a mutation in the ion channel gene KCNJ2 encoding the inward rectifier $\mathrm{K}^{+}$channel Kir2.1. This channel represents a component of the inward rectifier IK1.IK1 that, during the final phase of repolarization, provides repolarizing current. ${ }^{55}$

The ${ }^{23} \mathrm{Na}$ concentration in the skeletal using ${ }^{23} \mathrm{Na} \mathrm{MRI}$ operating at 7-T was higher in Cav1.1-R1239H, Cav1.1$\mathrm{R} 528 \mathrm{H}$, and Kir2.1 mutations in patients presenting with periodic paralysis compared with healthy volunteers. ${ }^{22}$ In this particular study, ${ }^{23} \mathrm{Na}$ and ${ }^{35} \mathrm{Cl} \mathrm{MR}$ examinations of both lower legs were performed on a 7-T whole-body system in genetically confirmed HypoPP (Cav1.1-R1239H mutation, $n=5$; Cav1.1-R528H mutation, $n=8)$, ATS $(n=3)$, and 16 healthy volunteers. ${ }^{22}$ Muscle edema was assessed on short tau inversion recovery (STIR) $3-\mathrm{T}^{1} \mathrm{H}$ MR images obtained the same day, fat replacement was measured on T1-weighted images, and the MFF was quantified using Dixon-type imaging. ${ }^{22}$ Median muscular ${ }^{23} \mathrm{Na}$ concentration was significantly higher in Cav1.1-R1239H $(35 \mathrm{mmol} / \mathrm{L})$, Cav1.1-R528H (32 $\mathrm{mmol} / \mathrm{L})$, and Kir2.1 mutation $(24 \mathrm{mmol} / \mathrm{L})$ than in healthy volunteers $(20 \mathrm{mmol} / \mathrm{L}){ }^{22}$ Median muscular normalized ${ }^{35} \mathrm{Cl}$ signal intensity detected by $7-\mathrm{T}{ }^{35} \mathrm{Cl}$ MRI was significantly higher in Cav1.1-R1239H (28), Cav1.1-R528H (24), but not in Kir2.1 mutations (14) of patients with periodic paralysis than in healthy volunteers (13) (-Fig. 3). Also, a strong correlation was found between the degree of muscular edema and MFF when compared with the muscular ${ }^{23} \mathrm{Na}$ concentration and normalized ${ }^{35} \mathrm{Cl}$ signal intensity, thereby substantiating the link between ion overload and cell damage in muscle tissue of periodic paralyses. $^{22}$ Compared with volunteers, Cav1.1-R1239H and Cav1.1-R528H showed both significantly increased muscular edema and MFF. In addition, $\mathrm{Na}^{+}$and $\mathrm{Cl}^{-}$homeostasis was mostly altered in the severe phenotype Cav1.1-R1239H presenting with almost daily paralytic episodes. ${ }^{22}$

\section{Outlook: Imaging of More Exotic X-nuclei $\left({ }^{35} \mathrm{Cl}\right.$ and $\left.{ }^{39} \mathrm{~K}\right)$}

In addition to $\mathrm{Na}^{+}$, many other MR-accessible nuclei are involved in physiologic cellular processes. However, except for ${ }^{31} \mathrm{P}$, their MR sensitivities are much lower compared with the sensitivity of $\mathrm{Na}^{+}$( - Table $\mathbf{1}$ ).

${ }^{35} \mathrm{Cl}$ MRI: Chlorine $\left(\mathrm{Cl}^{-}\right)$is the most abundant anion in the human organism and very important because it is involved in many fundamental physiologic processes like the influence of cellular osmolarity and cell volume. ${ }^{35} \mathrm{Cl}^{-}$is passively distributed in response to the resting membrane potential of skeletal muscle tissue, and thus it regulates cell potential such as the inhibition of muscular cell excitability. ${ }^{61}$ This results from very high $\mathrm{Cl}^{-}$conductance that is $\sim 80 \%$ of the total membrane conductance at rest. ${ }^{37}$ Hence the resting membrane potential of muscle cells can be approximated by the Nernst equation for $\mathrm{Cl}^{-}$. This equation requires only intra- and extracellular $\mathrm{Cl}^{-}$ concentrations. ${ }^{59} \mathrm{C}^{-}$MRI can determine the total tissue $\mathrm{Cl}^{-}$ concentration and thus can help calculate the required intracellular concentration. The extracellular concentration is easily accessible by blood chemistry, and data for the intracellular volume fraction are available in the literature ${ }^{62,63}$ and can also be estimated from the tissue sodium concentration (e.g., as measured with $\left.{ }^{23} \mathrm{Na} \mathrm{MRI}^{64}\right)$. Chlorine has two MR-sensitive naturally occurring isotopes, ${ }^{35} \mathrm{Cl}$ (natural abundancy, $\sim 76 \%$ ) and ${ }^{37} \mathrm{Cl}(\sim 24 \%) .{ }^{55}$ The feasibility of ${ }^{35} \mathrm{Cl}$ MRI in humans was demonstrated. ${ }^{22,59} \mathrm{~A}{ }^{35} \mathrm{Cl}$ MRI might enable noninvasive electrophysiologic measurements in vivo in the future, such as the determination of the resting membrane potential of muscle cells that is reduced in various muscular diseases including the periodic paralysis.

${ }^{39} \mathrm{~K}$ MRI: Because intra- and extracellular $\mathrm{K}^{+}$concentrations largely affect the excitability and membrane potential of cells, ${ }^{65}$ the measurement of the muscular $\mathrm{K}^{+}$content is highly preferable. Among the three naturally occurring MRsensitive isotopes of potassium $\left({ }^{39} \mathrm{~K},{ }^{40} \mathrm{~K}\right.$, and $\left.{ }^{41} \mathrm{~K}\right)$, the abundance of ${ }^{39} \mathrm{~K}$ is highest, $\sim 93 \% .{ }^{55}$ Although the expected 

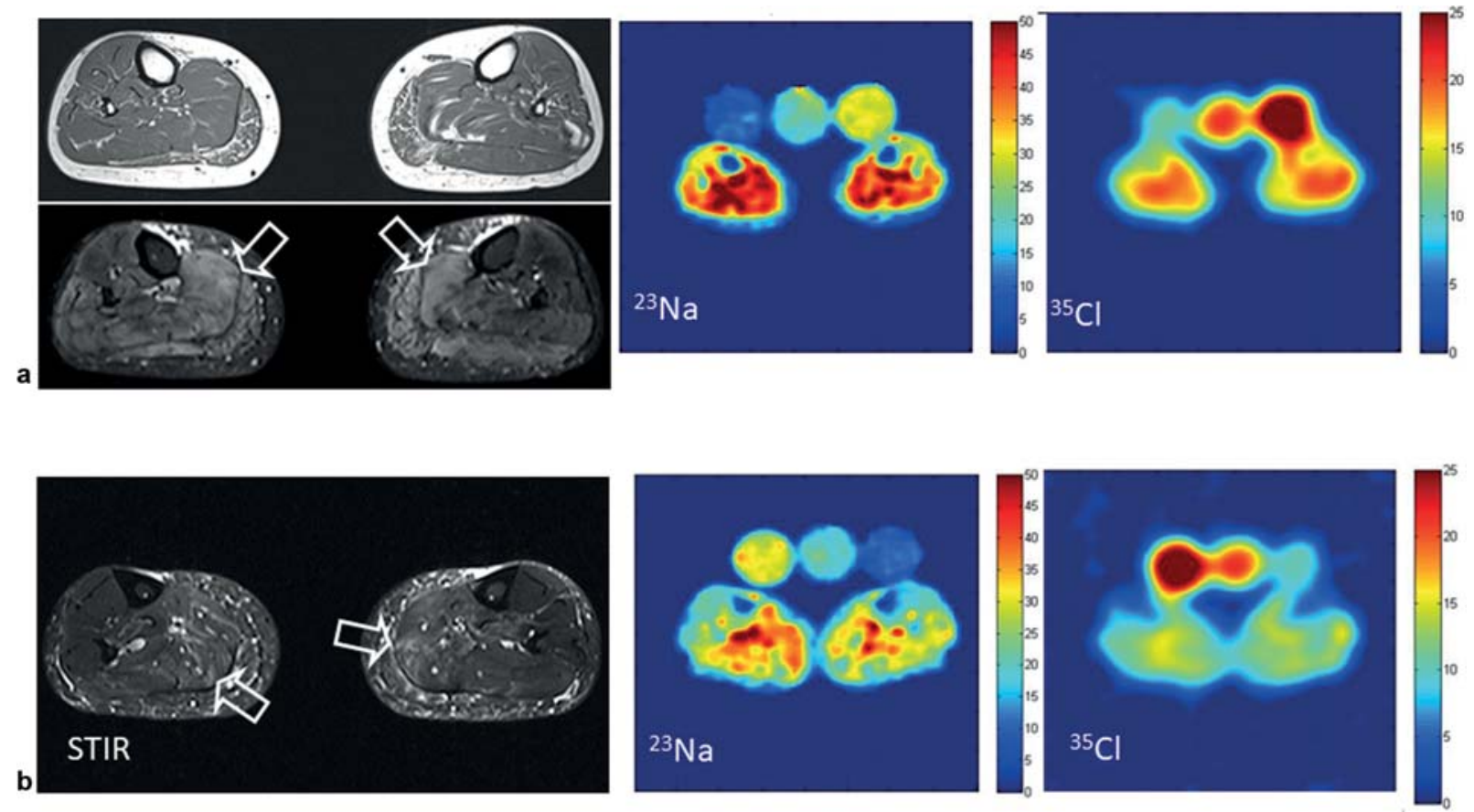

Fig. 3 Magnetic resonance imaging (MRI) findings in the two hypokalemic periodic paralysis (HypoPP) mutations. (a) A 41-year-old woman with HypoPP (severe phenotype, Cav1.1-R1239H) in comparison with (b) a 54-year-old woman with HypoPP (mild phenotype, Cav1.1-R528H). The higher muscular ${ }^{23} \mathrm{Na}$ and ${ }^{35} \mathrm{Cl}$ signals in the calf muscles in the severe phenotype Cav1.1-R1239H of HypoPP when compared with Cav1.1-R528H are evident. The three reference phantoms containing $5 \%$ agarose gel and 10,20 , or $30 \mathrm{mM} \mathrm{NaCl}$ solutions are visible on top of the calves. Both edema-like changes (open arrows) and fatty muscular degeneration within the calf muscles are most pronounced in Cav1.1-R1239H mutations (a). STIR, short tau inversion recovery.

Table 1 Physical properties and expected relative in vivo SNR of selected non-proton nuclei ${ }^{33,56}$

\begin{tabular}{|l|l|l|l|l|}
\hline Nucleus & Spin I & $\begin{array}{l}\text { Natural } \\
\text { abundance, } \\
\%\end{array}$ & $\begin{array}{l}\text { Typical in vivo } \\
\text { concentrations, } \\
\text { mol/L }\end{array}$ & $\begin{array}{l}\text { Relative in } \\
\text { vivo SNR, \% }\end{array}$ \\
\hline${ }^{1} \mathrm{H}$ & $1 / 2$ & 99.99 & $79^{\mathrm{b}}$ & 100 \\
\hline${ }^{23} \mathrm{Na}$ & $3 / 2$ & 100 & $0.17^{\mathrm{c}}$ & $\begin{array}{l}0.7 \cdot 10^{-2} / \\
1.3 \cdot 10^{-1}\end{array}$ \\
\hline${ }^{35} \mathrm{Cl}$ & $3 / 2$ & 75.78 & $0.03^{\mathrm{d}}$ & $2.2 \cdot 10^{-3}$ \\
\hline${ }^{39} \mathrm{~K}$ & $3 / 2$ & 93 & $0.11^{\mathrm{e}}$ & $1.6 \cdot 10^{-3}$ \\
\hline
\end{tabular}

Abbreviation: SNR, signal-to-noise ratio.

${ }^{\mathrm{a}} \mathrm{A}$ linear increase of noise with frequency was assumed.

berived from measured water content (71\%) of brain white matter. ${ }^{57}$

${ }^{\mathrm{c}}$ Measured ${ }^{23} \mathrm{Na}$ concentration of healthy calf muscle tissue ${ }^{36}$ and healthy articular cartilage ${ }^{58}$ (highest ${ }^{23} \mathrm{Na}$ content among all tissues). ${ }^{\mathrm{d}}$ Measured ${ }^{35} \mathrm{Cl}$ concentration of healthy brain white matter. ${ }^{59}$

${ }^{\mathrm{e}}$ Measured ${ }^{39} \mathrm{~K}$ concentration of skeletal muscle tissue. ${ }^{60}$

relative SNR of ${ }^{39} \mathrm{~K}$ MRI is more than a factor of 30 lower compared with the SNR of ${ }^{23} \mathrm{Na}$ MRI, the feasibility of ${ }^{35} \mathrm{Cl}$ and ${ }^{39} \mathrm{~K}$ MRI of human brain and muscle in vivo was demonstrated on a 7-T wb-MRI system (- Fig. 4)..$^{59,60}$

These non-proton qMRI techniques could be especially interesting for assessing non-dystrophic muscles, such as in periodic paralyses with often normal ${ }^{1} \mathrm{H}$ MRI findings, but also in DMD for detecting altered ion homeostasis in dystrophic muscle cells..$^{23,38,49}$ The recently introduced algorithm for partial volume correction leads to more accurate quantitative values in non-proton MRI, ${ }^{66}$ and hence the future for non-proton MRI in muscular applications may be even brighter. ${ }^{35}$

\section{Other Techniques: Diffusion Tensor Imaging/ Diffusion-weighted Imaging, Dynamic Contrast- enhanced MRI, and ${ }^{31} \mathrm{P}$ Magnetic Resonance Spectroscopy}

In addition to non-proton MRI, several other advanced imaging techniques have been used to study muscle diseases. Many of these were discussed in recent reviews, ${ }^{13,15,16,67,68}$ and therefore the information here is limited to a short summary of the technique and recent advances. The reviews offer more in-depth information.

Diffusion-weighted imaging (DWI) and DTI of muscle is a technique that allows for probing tissue microarchitecture. DWI with the increase in apparent diffusion coefficient (ADC) corresponds to the increase of extracellular water. ${ }^{13,69}$ DTI combined with appropriate postprocessing allows for determination of fiber lengths, fiber curvatures, and pennation angles. ${ }^{13}$ Although the application of this technique in the brain is widely used, the application in muscle disease is very much under development. Previous reports on human subjects showed changes in ADC due to muscle denervation $^{70}$ and in inflammatory myopathies. ${ }^{71}$ Stringent quality control and accurate fat suppression are of very high importance because both an increased $\mathrm{T} 2$ relaxation time and the presence of fat can influence the resulting values. ${ }^{72}$ Possibly as a result, there have been some conflicting reports on the changes reported in different muscle diseases. ${ }^{73-76}$ Overall, 
${ }^{1} \mathrm{H}$ MRI (T1 TSE)

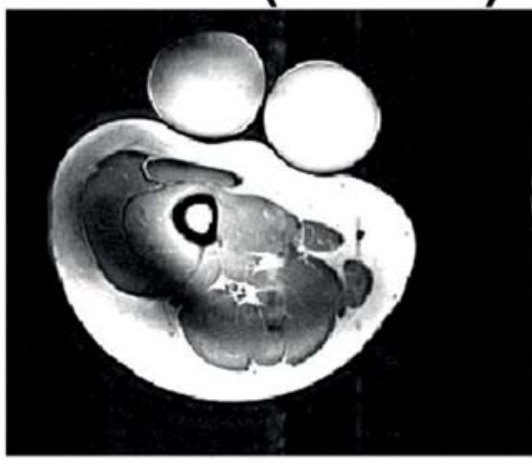

${ }^{39} \mathrm{~K}$ MRI

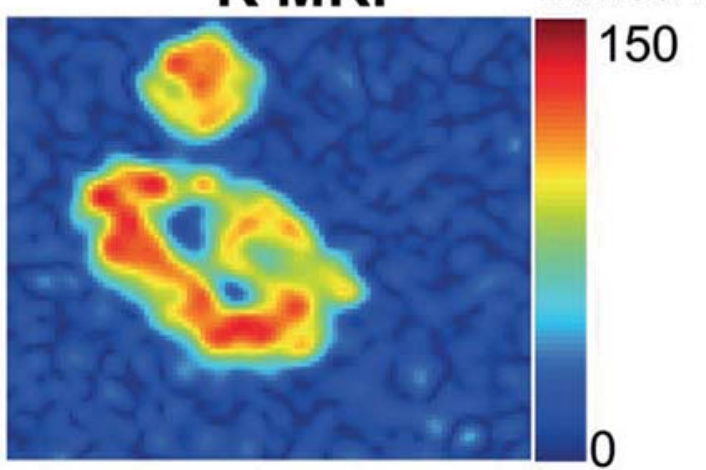

${ }^{23} \mathrm{Na}$ MRI

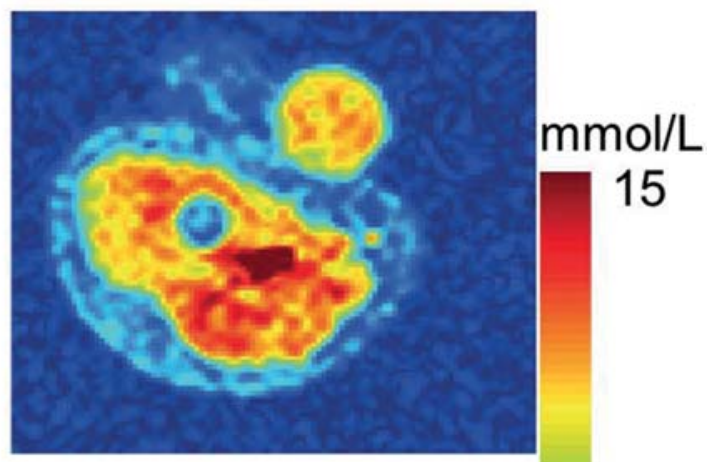

${ }^{35} \mathrm{CI}$ MRI

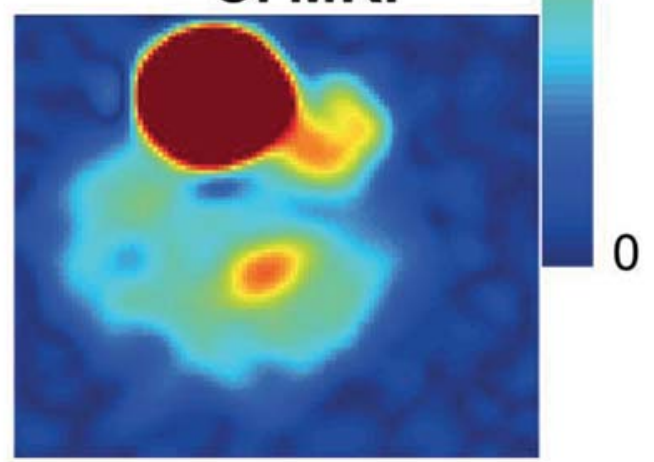

Fig. $4 \mathrm{~A}{ }^{1} \mathrm{H},{ }^{23} \mathrm{Na},{ }^{39} \mathrm{~K}$, and ${ }^{35} \mathrm{Cl}$ 7-T magnetic resonance imaging (MRI) of the healthy thigh muscle of a female volunteer. Each X-nuclei MR image was acquired in an acquisition time of 10 minutes. Nominal spatial resolutions of $3.8 \times 3.8 \times 10 \mathrm{~mm}^{3}\left({ }^{23} \mathrm{Na} \mathrm{MRI}\right), 8 \times 8 \times 16 \mathrm{~mm}{ }^{3}\left({ }^{39} \mathrm{~K} \mathrm{MRI}\right)$, and $12 \times 12 \times 24 \mathrm{~mm}^{3}\left({ }^{35} \mathrm{Cl} \mathrm{MRI}\right)$ were achieved. TSE, turbo spin echo. (Reproduced from Nagel et al. ${ }^{35}$ )

because changes in ADC are unspecific, the range in normal values is quite broad, ${ }^{13}$ and technical challenges are increased in pathologic conditions, the widespread application and interpretation of this technique is still challenging. A possible promising development could be using longer diffusion times with stimulated echo approaches because this allows modeling of membrane permeability and myofiber size, as recently shown in mouse models of DMD. ${ }^{77,78}$

Current magnetic resonance perfusion MRI techniques allowing the noninvasive functional assessment of peripheral microvasculature in healthy and diseased individuals are blood oxygenation level-dependent (BOLD), arterial spin labeling (ASL), and dynamic contrast-enhanced (DCE) MRI. The functional imaging of skeletal muscle microvasculature helps in the understanding of muscular and vascular physiology and alterations of microcirculation under certain pathologic conditions such as peripheral arterial occlusive disease, diabetes mellitus, chronic compartment syndrome, and rheumatic disorders.

The potential of these three MRI methods to assess disease severity noninvasively and the efficacy of new therapeutic strategies such as stem cell and gene therapy renders them very appealing future research targets. ${ }^{79}$ Furthermore, denervated musculature shows gadolinium enhancement that can be detected $\sim 24$ hours after the denervating event. ${ }^{80} \mathrm{MR}$ perfusion imaging is capable of detecting increased perfusion in denervated muscles compared with normal muscu- lature. $^{81}$ When no reinnervation occurs, chronic muscle denervation finally results in atrophy, with reduction of the diameter of the muscle belly and fatty metaplasia leading to a shortening of the $\mathrm{T} 1$ relaxation time.

MRS and magnetic resonance spectroscopic imaging (MRSI) provide metabolic information on the musculoskeletal system, thus helping us understand the biochemical and pathophysiologic nature of numerous diseases. In particular, 31-phosphorous $\left({ }^{31} \mathrm{P}\right)$ MRS was used to study the energy metabolism of muscular tissue since the very beginning of MR examinations in humans when small-bore magnets for studies of the limbs became available. ${ }^{67,82}$ Spatial localization was less demanding in these studies. However, high temporal resolution was necessary to follow metabolism during exercise and recovery. The observation of high-energy phosphates during and after the application of workload offers insight into oxidative phosphorylation, a process that takes place in the mitochondria and characterizes impaired mitochondrial function. ${ }^{83}$ In healthy skeletal muscle tissue, the amount of high-energy phosphates and the oxidativephosphorylation potential are crucial for proper muscle function. Disturbances in the kinetics of energy metabolism, such as oxidative phosphorylation during recovery from exercise, may limit the contracting ability of the muscles. ${ }^{82}$ Furthermore, changes in myocellular high-energy phosphate metabolism are encountered in diseases leading to muscular degeneration. ${ }^{13,82}$ Intracellular $\mathrm{pH}$, as well as the MR signal 
intensity ratios $\mathrm{P}_{\mathrm{i}}: \mathrm{PCr}$ (inorganic phosphate $\left[\mathrm{P}_{\mathrm{i}}\right.$ to phosphocreatine $[\mathrm{PCr}]$ ) and $\mathrm{PDE}: \mathrm{PCr}$ (phosphodiester [PDE] to $\mathrm{PCr}$ ), were reported to increase depending on the stage and severity of the muscle degeneration. ${ }^{13,67,68,82}$ It was shown conclusively how MRS and MRSI were used in numerous diseases to characterize an involvement of the muscular metabolism. Recent reviews offer more in-depth information. ${ }^{13,67,68}$

\section{Conclusion}

The importance of muscle imaging in patients with muscular disorders is increasingly recognized. Today muscle MR imaging with its capability of whole-body musculature assessment is the modality of choice in a routine diagnostic work-up, often able to identify a distinctive pattern of muscle involvement. In the future, wb-MRI protocols and hierarchical meta-analytical approaches with fingerprints of individual diseases will probably very much improve the use of muscle MRI for pattern recognition purposes. Quantitative MRI may be a useful tool for monitoring disease progression, especially when subclinical, in future therapeutic trials. In addition, advanced imaging technologies such as non-proton MRI, DTI, perfusion MRI, T2 mapping, and MRS/MRSI will become more available, at least in an university hospital setting, to reveal deeper insights into muscle pathophysiology beyond the mere detection of fatty or edema-like changes.

\section{Conflict of Interest}

Mike P. Wattjes has received speaking and consultancy fees from Biogen, IXICO, Novartis, Roche, Sanofi-Genzyme, and Springer Healthcare. He has received royalties from Springer for the book Neuromuscular Imaging. Hermien E. Kan reports research support from Philips Healthcare, consultancy for PTC Therapeutics, and trial support from ImagingDMD-UF outside the submitted work. Armin M. Nagel reports research support from Siemens Healthineers AG. All the authors have declared no conflicts of interest for this article.

\section{References}

1 Wattjes MP, Fischer D, eds. Neuromuscular Imaging. New York, NY: Springer Science+Business Media; 2013

2 Paoletti M, Pichiecchio A, Cotti Piccinelli S, et al. Advances in quantitative imaging of genetic and acquired myopathies: clinical applications and perspectives. Front Neurol 2019;10:78

3 Hobson-Webb LD. Emerging Technologies in Neuromuscular Ultrasound. Muscle Nerve 2020; February 3; (Epub ahead of print)

4 Wattjes MP, Kley RA, Fischer D. Neuromuscular imaging in inherited muscle diseases. Eur Radiol 2010;20(10):2447-2460

5 Díaz-Manera J, Llauger J, Gallardo E, Illa I. Muscle MRI in muscular dystrophies. Acta Myol 2015;34(2-3):95-108

6 Ten Dam L, van der Kooi AJ, Verhamme C, Wattjes MP, de Visser M. Muscle imaging in inherited ad acquired muscle diseases. Eur J Neurol 2016;23(04):688-703

7 Wattjes MP, Fischmann A, Fischer D. Imaging of primary muscular diseases: What do neurologists expect from radiologists? [in German]. Radiologe 2017;57(12):1005-1011
8 Warman Chardon J, Díaz-Manera J, Tasca G, et al; MYO-MRI Working Group. MYO-MRI diagnostic protocols in genetic myopathies. Neuromuscul Disord 2019;29(11):827-841

9 Leung DG. Magnetic resonance imaging patterns of muscle involvement in genetic muscle diseases: a systematic review. J Neurol 2017;264(07):1320-1333

10 Kornblum C, Lutterbey GG, Czermin B, et al. Whole-body highfield MRI shows no skeletal muscle degeneration in young patients with recessive myotonia congenita. Acta Neurol Scand 2010;121(02):131-135

11 Kesper K, Kornblum C, Reimann J, Lutterbey G, Schröder R, Wattjes MP. Pattern of skeletal muscle involvement in primary dysferlinopathies: a whole-body 3.0-T magnetic resonance imaging study. Acta Neurol Scand 2009;120(02):111-118

12 Weber MA, Wolf M, Wattjes MP. Imaging patterns of muscle atrophy. Semin Musculoskelet Radiol 2018;22(03):299-306

13 Strijkers GJ, Araujo ECA, Azzabou N, et al. Exploration of new contrasts, targets, and MR imaging and spectroscopy techniques for neuromuscular disease. A Workshop Report of Working Group 3 of the Biomedicine and Molecular Biosciences COST Action BM1304 MYO-MRI. J Neuromuscul Dis 2019;6(01):1-30

14 Damon BM, Li K, Bryant ND. Magnetic resonance imaging of skeletal muscle disease. Handb Clin Neurol 2016;136:827-842

15 Carlier PG, Marty B, Scheidegger O, et al. Skeletal muscle quantitative nuclear magnetic resonance imaging and spectroscopy as an outcome measure for clinical trials. J Neuromuscul Dis 2016;3(01):1-28

16 Leung DG. Advancements in magnetic resonance imaging-based biomarkers for muscular dystrophy. Muscle Nerve 2019;60(04): 347-360

17 Fischmann A, Hafner P, Fasler S, et al. Quantitative MRI can detect subclinical disease progression in muscular dystrophy. J Neurol 2012;259(08):1648-1654

18 Figueroa-Bonaparte S, Llauger J, Segovia S, et al; Spanish Pompe group. Quantitative muscle MRI to follow up late onset Pompe patients: a prospective study. Sci Rep 2018;8(01):10898

19 Regula JU, Jestaedt L, Jende F, Bartsch A, Meinck HM, Weber MA. Clinical muscle testing compared with whole-body magnetic resonance imaging in facio-scapulo-humeral muscular dystrophy. Clin Neuroradiol 2016;26(04):445-455

20 Ma J. Dixon techniques for water and fat imaging. J Magn Reson Imaging 2008;28(03):543-558

21 Burakiewicz J, Sinclair CDJ, Fischer D, Walter GA, Kan HE, Hollingsworth KG. Quantifying fat replacement of muscle by quantitative MRI in muscular dystrophy. J Neurol 2017;264(10):2053-2067

22 Weber MA, Nagel AM, Marschar AM, et al. 7-T (35)Cl and (23) Na MR imaging for detection of mutation-dependent alterations in muscular edema and fat fraction with sodium and chloride concentrations in muscular periodic paralyses. Radiology 2016; 280(03):848-859

23 Fischer D, Bonati U, Wattjes MP. Recent developments in muscle imaging of neuromuscular disorders. Curr Opin Neurol 2016;29 (05):614-620

24 Bonati U, Schmid M, Hafner P, et al. Longitudinal 2-point Dixon muscle magnetic resonance imaging in Becker muscular dystrophy. Muscle Nerve 2015;51(06):918-921

25 Morrow JM, Sinclair CDJ, Fischmann A, et al. MRI biomarker assessment of neuromuscular disease progression: a prospective observational cohort study. Lancet Neurol 2016;15(01):65-77

26 Willcocks RJ, Rooney WD, Triplett WT, et al. Multicenter prospective longitudinal study of magnetic resonance biomarkers in a large Duchenne muscular dystrophy cohort. Ann Neurol 2016;79 (04):535-547

27 Dahlqvist JR, Oestergaard ST, Poulsen NS, Knak KL, Thomsen C, Vissing J. Muscle contractility in spinobulbar muscular atrophy. Sci Rep 2019;9(01):4680

28 Murphy AP, Morrow J, Dahlqvist JR, et al. Natural history of limb girdle muscular dystrophy R9 over 6 years: searching for trial endpoints. Ann Clin Transl Neurol 2019;6(06):1033-1045 
29 Barnard AM, Willcocks RJ, Triplett WT, et al. MR biomarkers predict clinical function in Duchenne muscular dystrophy. Neurology 2020;94(09):e897-e909

30 Naarding KJ, Reyngoudt H, van Zwet EW, et al. MRI vastus lateralis fat fraction predicts loss of ambulation in Duchenne muscular dystrophy. Neurology 2020;94(13):e1386-e1394

31 Straub V, Balabanov P, Bushby K, et al. Stakeholder cooperation to overcome challenges in orphan medicine development: the example of Duchenne muscular dystrophy. Lancet Neurol 2016;15 (08):882-890

32 Ladd ME, Bachert P, Meyerspeer M, et al. Pros and cons of ultrahigh-field MRI/MRS for human application. Prog Nucl Magn Reson Spectrosc 2018;109:1-50

33 Niesporek SC, Nagel AM, Platt T. Multinuclear MRI at ultrahigh fields. Top Magn Reson Imaging 2019;28(03):173-188

34 Madelin G, Regatte RR. Biomedical applications of sodium MRI in vivo. J Magn Reson Imaging 2013;38(03):511-529

35 Nagel AM, Weber MA, Borthakur A, Reddy R. Skeletal muscle MR imaging beyond protons: with a focus on sodium MRI in musculoskeletal applications. In: Weber MA, ed. Magnetic Resonance Imaging of the Skeletal Musculature. New York, NY: Springer; 2014:115-133

36 Gerhalter T, Gast LV, Marty B, Uder M, Carlier PG, Nagel AM. Assessing the variability of ${ }^{23} \mathrm{Na}$ MRI in skeletal muscle tissue: reproducibility and repeatability of tissue sodium concentration measurements in the lower leg at 3 T. NMR Biomed 2020;33(05): e4279

37 Lehmann-Horn F, Jurkat-Rott K. Voltage-gated ion channels and hereditary disease. Physiol Rev 1999;79(04):1317-1372

38 Jurkat-Rott K, Weber MA, Lehmann-Horn F. MRI in muscle channelopathies. In: Weber MA, ed. Magnetic Resonance Imaging of the Skeletal Musculature. New York, NY: Springer; 2014:271-288

39 Kornblum C, Lutterbey G, Bogdanow M, et al. Distinct neuromuscular phenotypes in myotonic dystrophy types 1 and $2:$ a whole body highfield MRI study. J Neurol 2006;253(06):753-761

40 Nagel AM, Amarteifio E, Lehmann-Horn F, et al. 3 Tesla sodium inversion recovery magnetic resonance imaging allows for improved visualization of intracellular sodium content changes in muscular channelopathies. Invest Radiol 2011;46(12):759-766

41 Jurkat-Rott K, Lehmann-Horn F, Elbaz A, et al. A calcium channel mutation causing hypokalemic periodic paralysis. Hum Mol Genet 1994;3(08):1415-1419

42 Jurkat-Rott K, Weber MA, Fauler M, et al. $\mathrm{K}^{+}$-dependent paradoxical membrane depolarization and $\mathrm{Na}^{+}$overload, major and reversible contributors to weakness by ion channel leaks. Proc Natl Acad Sci U S A 2009;106(10):4036-4041

43 Fan C, Lehmann-Horn F, Weber MA, et al. Transient compartmentlike syndrome and normokalaemic periodic paralysis due to a $\mathrm{Ca}$ (v)1.1 mutation. Brain 2013;136(Pt 12):3775-3786

44 Resnick JS, Engel WK, Griggs RC, Stam AC. Acetazolamide prophylaxis in hypokalemic periodic paralysis. N Engl J Med 1968;278 (11):582-586

45 Weber MA, Nagel AM, Jurkat-Rott K, Lehmann-Horn F. Sodium (23Na) MRI detects elevated muscular sodium concentration in Duchenne muscular dystrophy. Neurology 2011;77(23):2017-2024

46 Amarteifio E, Nagel AM, Weber MA, Jurkat-Rott K, Lehmann-Horn F. Hyperkalemic periodic paralysis and permanent weakness: 3-T MR imaging depicts intracellular 23Na overload-initial results. Radiology 2012;264(01):154-163

47 Chang G, Wang L, Schweitzer ME, Regatte RR III. 3D 23Na MRI of human skeletal muscle at 7 Tesla: initial experience. Eur Radiol 2010;20(08):2039-2046

48 Weber MA, Jurkat-Rott K, Lerche H, Lehmann-Horn F. Strength and muscle structure preserved during long-term therapy in a patient with hypokalemic periodic paralysis (Cav1.1-R1239G). J Neurol 2019;266(07):1623-1632

49 Weber MA, Nagel AM, Wolf MB, et al. Permanent muscular sodium overload and persistent muscle edema in Duchenne muscular dystrophy: a possible contributor of progressive muscle degeneration. J Neurol 2012;259(11):2385-2392

50 Glemser PA, Jaeger H, Nagel AM, et al. ${ }^{23} \mathrm{Na}$ MRI and myometry to compare eplerenone vs. glucocorticoid treatment in Duchenne dystrophy. Acta Myol 2017;36(01):2-13

51 Gerhalter T, Gast LV, Marty B, et al. ${ }^{23} \mathrm{Na}$ MRI depicts early changes in ion homeostasis in skeletal muscle tissue of patients with Duchenne muscular dystrophy. J Magn Reson Imaging 2019;50 (04):1103-1113

52 Manzur AY, Kuntzer T, Pike M, Swan A. Glucocorticoid corticosteroids for Duchenne muscular dystrophy. Cochrane Database Syst Rev 2008;(01):CD003725

53 Lehmann-Horn F, Weber MA, Nagel AM, et al. Rationale for treating oedema in Duchenne muscular dystrophy with eplerenone. Acta Myol 2012;31(01):31-39

54 Statland JM, Barohn RJ. Muscle channelopathies: the nondystrophic myotonias and periodic paralyses. Continuum (Minneap Minn) 2013;19(6 Muscle Disease):1598-1614

55 Tristani-Firouzi M, Etheridge SP. Kir 2.1 channelopathies: the Andersen-Tawil syndrome. Pflugers Arch 2010;460(02):289-294

56 Harris RK, Becker ED, Cabral de Menezes SM, Goodfellow R, Granger P. NMR nomenclature: nuclear spin properties and conventions for chemical shifts. IUPAC Recommendations 2001. Solid State Nucl Magn Reson 2002;22(04):458-483

57 Neeb H, Zilles K, Shah NJ. A new method for fast quantitative mapping of absolute water content in vivo. Neuroimage 2006;31 (03):1156-1168

58 Madelin G, Lee JS, Regatte RR, Jerschow A. Sodium MRI: methods and applications. Prog Nucl Magn Reson Spectrosc 2014; 79:14-47

59 Nagel AM, Lehmann-Horn F, Weber MA, et al. In vivo 35Cl MR imaging in humans: a feasibility study. Radiology 2014;271(02): 585-595

60 Umathum R, Roesler MB, Nagel AM. In vivo 39K MR imaging of human muscle and brain. Radiology 2013;269(02):569-576

61 Koch MC, Steinmeyer K, Lorenz C, et al. The skeletal muscle chloride channel in dominant and recessive human myotonia. Science 1992;257(5071):797-800

62 Donahue KM, Weisskoff RM, Parmelee DJ, et al. Dynamic Gd-DTPA enhanced MRI measurement of tissue cell volume fraction. Magn Reson Med 1995;34(03):423-432

63 Syková E, Nicholson C. Diffusion in brain extracellular space. Physiol Rev 2008;88(04):1277-1340

64 Thulborn KR, Gindin TS, Davis D, Erb P. Comprehensive MR imaging protocol for stroke management: tissue sodium concentration as a measure of tissue viability in nonhuman primate studies and in clinical studies. Radiology 1999;213(01):156-166

65 Hodgkin AL, Huxley AF. A quantitative description of membrane current and its application to conduction and excitation in nerve.J Physiol 1952;117(04):500-544

66 Niesporek SC, Umathum R, Fiedler TM, Bachert P, Ladd ME, Nagel AM. Improved $T^{*}$ determination in ${ }^{23} \mathrm{Na},{ }^{35} \mathrm{Cl}$, and ${ }^{17} \mathrm{O}$ MRI using iterative partial volume correction based on ${ }^{1} \mathrm{H}$ MRI segmentation. MAGMA 2017;30(06):519-536

67 Boesch C. MR spectroscopy and spectroscopic imaging for evaluation of skeletal muscle metabolism: basics and applications in metabolic diseases. In: Weber MA, ed. Magnetic Resonance Imaging of the Skeletal Musculature. New York, NY: Springer; 2014:135-163

68 Valkovič L, Chmelík M, Krššák M. In-vivo ${ }^{31}$ P-MRS of skeletal muscle and liver: a way for non-invasive assessment of their metabolism. Anal Biochem 2017;529:193-215

69 Oudeman J, Nederveen AJ, Strijkers GJ, Maas M, Luijten PR, Froeling M. Techniques and applications of skeletal muscle diffusion tensor imaging: a review. J Magn Reson Imaging 2016;43(04):773-788

70 Holl N, Echaniz-Laguna A, Bierry G, et al. Diffusion-weighted MRI of denervated muscle: a clinical and experimental study. Skeletal Radiol 2008;37(12):1111-1117 
71 Qi J, Olsen NJ, Price RR, Winston JA, Park JH. Diffusion-weighted imaging of inflammatory myopathies: polymyositis and dermatomyositis. J Magn Reson Imaging 2008;27(01):212-217

72 Hooijmans MT, Damon BM, Froeling M, et al. Evaluation of skeletal muscle DTI in patients with Duchenne muscular dystrophy. NMR Biomed 2015;28(11):1589-1597

73 Maggi L, Moscatelli M, Frangiamore R, et al. Quantitative muscle MRI protocol as possible biomarker in Becker muscular dystrophy. Clin Neuroradiol 2020; January 23 ( Epub ahead of print)

74 Rehmann R, Schlaffke L, Froeling M, et al. Muscle diffusion tensor imaging in glycogen storage disease $\mathrm{V}$ (McArdle disease). Eur Radiol 2019;29(06):3224-3232

75 Li GD, Liang YY, Xu P, Ling J, Chen YM. Diffusion-tensor imaging of thigh muscles in Duchenne muscular dystrophy: correlation of apparent diffusion coefficient and fractional anisotropy values with fatty infiltration. AJR Am J Roentgenol 2016;206(04):867-870

76 Ponrartana S, Ramos-Platt L, Wren TA, et al. Effectiveness of diffusion tensor imaging in assessing disease severity in Duchenne muscular dystrophy: preliminary study. Pediatr Radiol 2015;45(04):582-589

77 Winters KV, Reynaud O, Novikov DS, Fieremans E, Kim SG. Quantifying myofiber integrity using diffusion MRI and random permeable barrier modeling in skeletal muscle growth and
Duchenne muscular dystrophy model in mice. Magn Reson Med 2018;80(05):2094-2108

78 Porcari P, Hall MG, Clark CA, Greally E, Straub V, Blamire AM. Time-dependent diffusion MRI as a probe of microstructural changes in a mouse model of Duchenne muscular dystrophy. NMR Biomed 2020;33(05):e4276

79 Partovi S, Jacobi B, Gordon Y, et al. Assessment of skeletal muscle microperfusion using MRI. In: Weber MA, ed. Magnetic Resonance Imaging of the Skeletal Musculature. New York, NY: Springer; 2014:87-114

80 Bendszus M, Koltzenburg M. Visualization of denervated muscle by gadolinium-enhanced MRI. Neurology 2001;57(09):1709-1711

81 Goyault G, Bierry G, Holl N, et al. Diffusion-weighted MRI, dynamic susceptibility contrast MRI and ultrasound perfusion quantification of denervated muscle in rabbits. Skeletal Radiol 2012;41(01):33-40

82 Argov Z, Löfberg M, Arnold DL. Insights into muscle diseases gained by phosphorus magnetic resonance spectroscopy. Muscle Nerve 2000;23(09):1316-1334

83 Kemp GJ, Taylor DJ, Thompson CH, et al. Quantitative analysis by 31P magnetic resonance spectroscopy of abnormal mitochondrial oxidation in skeletal muscle during recovery from exercise. NMR Biomed 1993;6(05):302-310 